\title{
A simple characterization of principal ideal domains
}

\author{
by
}

Clifford S. Queen (Bethlehem, Pa.)

1. Introduction. In this note we give necessary and sufficient conditions for an integral domain to be a principal ideal domain. Curiously, these conditions are similar to those that characterize Euclidean domains. In Section 2 we establish notation, discuss related results and prove our theorem. Finally, in Section 3 we give two nontrivial applications to real quadratic number fields.

2. Results. Let $R$ be an integral domain and $K$ its field of fractions. We say that $R$ is a principal ideal domain (abbreviated P.I.D.) if every ideal of $R$ is principal. That is to say, given an ideal $\vartheta$ of $R$ there exists $\beta$ in $R$ such that $\vartheta=(\beta)=\beta R$. A necessary condition for $R$ to be a P.I.D. is that it be factorial or, in other words, that every nonzero element of $R$ can be written uniquely as a product of irreducible elements of $R$. But being factorial is not sufficient since the polynomial ring in one variable over the integers is factorial but not a P.I.D. (see [2]). A sufficient condition for $R$ to be a P.I.D. is that $R$ be Euclidean (see [7]). We mean by this that there is a map $N: R \rightarrow \mathbb{Z}^{*}$, where $\mathbb{Z}^{*}$ denotes the nonnegative integers, with the following properties:

(1) $N(\alpha)=0$ if and only if $\alpha=0$.

(2) Given $\alpha$ and $\beta$ in $R$ with $\beta \neq 0$ there exist $\theta$ and $\varrho$ in $R$ such that $\alpha=\beta \theta+\varrho$, with $0<N(\varrho)<N(\beta)$.

Familiar examples of Euclidean domains are the integers, the Gaussian integers and the polynomial ring in one variable over a field. In all of these examples one can choose a map $N$ with the following additional properties: $N(\alpha)=1$ if and only if $\alpha$ is a unit of $R$ and $N(\alpha \beta) \geq N(\beta)$ for all nonzero $\alpha$ and $\beta$ in $R$. It is known (see [7]) that there is no loss of generality in insisting on these additional properties. The ring $\mathbb{Z}[w]=\{x+y w \mid x, y$ in $\mathbb{Z}\}$, where 
$\mathbb{Z}$ denotes the ring of integers and $w=(1+\sqrt{-19}) / 2$ is an example of a P.I.D. which is not Euclidean (see [5]).

In the Theorem below we give necessary and sufficient conditions for $R$ to be a P.I.D. These conditions generalize properties discussed by Rabinowitsch and Kutsuna (see [3] and [6]). Recall that $\mathbb{Z}^{*}$ denotes the nonnegative integers and $\mathbb{Q}^{*}$ the nonnegative rational numbers.

TheOREM. Let $R$ be an integral domain and $K$ its field of fractions. Then $R$ is a P.I.D. if and only if there is a map $N: K \rightarrow \mathbb{Q}^{*}$ with the following properties:

(0) For all $\xi$ in $K, N(\xi)=0$ if and only if $\xi=0$;

(1) $N(R)$ is a subset of $\mathbb{Z}^{*}$;

(2) For elements $\alpha$ in $R, N(\alpha)=1$ if and only if $\alpha$ is a unit;

(3) For all $\xi$ and $\zeta$ in $K, N(\zeta \xi)=N(\zeta) N(\xi)$;

(4) For any $\xi$ in $K$ such that $\xi$ is not in $R$ there exist $\alpha$ and $\beta$ in $R$ with $0<N(\xi \alpha-\beta)<1$.

Proof. Suppose $R$ is a P.I.D. Our task will be to construct a map $N$ satisfying properties (0) through (4). Let $P$ be a set consisting of one irreducible element for each associate class of irreducible elements of $R$. If $\alpha$ is a nonzero element of $R$, let $v(\alpha)$ denote the number of irreducible factors of $\alpha$ taken from $P$ and counting multiplicity. For example, if $\left\{\pi_{1}, \pi_{2}, \ldots, \pi_{k}\right\}$ $\subset P$, then $v\left(\pi_{1}^{n_{1}} \pi_{2}^{n_{2}} \ldots \pi_{k}^{n_{k}}\right)=n_{1}+n_{2}+\ldots+n_{k}$. Since $R$ is factorial, it follows that the map $v: R-\{0\} \rightarrow \mathbb{Z}^{*}$ is well defined and enjoys the following two properties:

(i) $v(\alpha)=0$ if and only if $\alpha$ is a unit of $R$;

(ii) $v(\alpha \beta)=v(\alpha)+v(\beta)$ for all $\alpha$ and $\beta$ in $R-\{0\}$.

Consider the map $N: K \rightarrow \mathbb{Q}^{*}$ defined as follows: If $\xi$ is a nonzero element of $K$ then there exist $\alpha$ and $\beta$ in $R$ such that $\alpha \beta \neq 0$ and $\xi=\alpha / \beta$. Now define $N(\xi)=2^{v(\alpha)-v(\beta)}$ and set $N(0)=0$. Our map satisfies $(0)$ and (1) by definition and properties (2) and (3) are immediate from (i) and (ii).

We need to show that $N$ satisfies property (4). Let $\xi$ be an element of $K$ not in $R$. Then there exist $\alpha$ and $\beta$ in $R$ such that $\alpha \beta \neq 0$ and $\xi=\alpha / \beta$. Because $R$ is a P.I.D., there is a nonzero element $\delta$ in $R$ such that $(\delta)=\delta R=\alpha R+\beta R$. Since $\delta$ divides both $\alpha$ and $\beta$, we have $\beta=\delta \nu$, where $\nu$ is in $R$. Further, if $\nu$ were a unit of $R$, then $\beta$ would divide $\alpha$, contrary to our assumption. Thus $0<N(\delta)<N(\delta) N(\nu)=N(\beta)$. Finally, because there exist $\eta$ and $\gamma$ in $R$ such that $\delta=\alpha \eta-\beta \gamma$, we have $0<N(\xi \eta-\gamma)=$ $N(\delta / \beta)<1$.

Now suppose that $N$ is a map from $K$ to $\mathbb{Q}^{*}$ satisfying properties $(0)$ through (4). Let $\vartheta$ be a nonzero ideal of $R$ and choose $\beta$ in $\vartheta$ such that $N(\beta)$ is minimal over nonzero elements of $\vartheta$. If $N(\beta)=1$ then $\beta$ is a unit 
and $\vartheta=R$, a principal ideal. Next suppose that $N(\beta)>1$. We will show that $\vartheta=\beta R$ by showing that $\beta$ divides every element $\alpha$ in $\vartheta$. To that end, let $\alpha$ be in $\vartheta$ and assume that $\beta$ does not divide $\alpha$. Then it follows that $\xi=\alpha / \beta$ is in $K$ but not in $R$. So by property (4) there exist $\eta$ and $\gamma$ in $R$ with $0<N(\xi \eta-\gamma)<1$, i.e. $0<N(\alpha \eta-\beta)<N(\beta)$. Now since $\alpha \eta-\beta \gamma$ is a nonzero element of $\vartheta$, we have a contradiction to the minimality of $N(\beta)$.

Corollary. If $R$ is a P.I.D. and $N: K \rightarrow \mathbb{Q}^{*}$ satisfies properties (1) through (3) above, then property (4) is also satisfied.

Proof. This is just the first part of the proof of the Theorem.

3. Applications. To show that our necessary and sufficient condition is not impossible to use, we present easy proofs of two well known results.

Proposition. Let $p$ be a rational integer prime such that $p \equiv 5(\bmod 8)$. Consider the ring $R=\mathbb{Z}[\sqrt{2 p}]$. Then $R$ is not a P.I.D.

Proof. It is well known that $R$ is the ring of integers in $K=\mathbb{Q}(\sqrt{2 p})$ (see [1]). The absolute value of the norm map from $K$ to $\mathbb{Q}$ is given by $N(s+$ $t \sqrt{2 p})=\left|s^{2}-2 p t^{2}\right|$, where $s$ and $t$ are in $\mathbb{Q}$. Now it is easy to show that $N$ satisfies properties (0) through (3). So if $R$ were a P.I.D. then, by the above corollary and the fact that $\sqrt{2 p} / 2$ is not in $R$, there would exist $x+y \sqrt{2 p}$ and $z+w \sqrt{2 p}$ in $R$ such that $0<N(\sqrt{2 p}(x+y \sqrt{2 p})-2(z+w \sqrt{2 p}))=$ $\left|(2 p y-2 z)^{2}-2 p(x-2 w)^{2}\right|<4$. That is $0<\left|2(p y-z)^{2}-p(x-2 w)^{2}\right|<2$ and so $2(p y-z)^{2}-p(x-2 w)^{2}= \pm 1$. Therefore $2 z^{2} \equiv \pm 1(\bmod p)$ and by the assumption on $p$ this is impossible.

Proposition. Suppose $p$ is a rational integer prime with $p \equiv 5(\bmod 8)$ and $p>5$. Consider the ring $\mathbb{Z}[\omega]$, where $\omega=(1+\sqrt{5 p}) / 2$. Then $R$ is not a P.I.D.

Proof. Our map is the absolute value of the norm from $K=\mathbb{Q}(\omega)$ to $\mathbb{Q}$ given by $N(s+t \omega)=\left|s^{2}+s t-t^{2}(5 p-1) / 2\right|$, where $s$ and $t$ are in $\mathbb{Q}$. Since $\omega / 2$ is not in $R$, if $R$ were a P.I.D. there would exist elements $x+y \omega$ and $z+w \omega$ in $R$ such that

$$
0<N(\omega(x+y \omega)-2(z+w \omega))<4 .
$$

That is to say,

$$
\begin{aligned}
0<\mid(-2 z+y(5 p-1) / 4)^{2}+(-2 z+y & (5 p-1) / 4)(x+y-2 w) \\
- & (x+y-2 w)^{2}(5 p-1) / 4 \mid<4 .
\end{aligned}
$$

Now if $p=8 k+5$, then $(5 p-1) / 4=2(5 k+3)$ and thus

$2(-z+y(5 k+3))^{2}+(-z+y(5 k+3))(x+y-2 w)-(x+y-2 w)^{2}(5 k+3)= \pm 1$. 
Now setting $A=-z+y(5 k+3), B=x+y-2 w$ and computing modulo 5 we have

$$
2 A^{2}+A B-3 B^{2} \equiv 2(A-B)^{2} \equiv \pm 1(\bmod 5),
$$

which is clearly impossible.

\section{References}

[1] H. Cohn, Advanced Number Theory, Dover, 1980.

[2] N. Jacobson, Basic Algebra I, 2nd ed., Freeman, 1985.

[3] M. Kutsuna, On a criterion for the class number of a real quadratic field to be one, Nagoya Math. J. 79 (1980), 123-129.

[4] T. Motzkin, The Euclidean algorithm, Bull. Amer. Math. Soc. 55 (1949), 1142-1146.

[5] C. Queen, Arithmetic euclidean rings, Acta Arith. 26 (1974), 105-113.

[6] G. Rabinowitsch, Eindeutigkeit der Zerlegung in Primzahlfaktoren in quadratischen Zahlkörpern, J. Reine Angew. Math. 142 (1913), 153-164.

[7] P. Samuel, About Euclidean rings, J. Algebra 19 (1971), 282-301.

DEPARTMENT OF MATHEMATICS

LEHIGH UNIVERSITY

BETHLEHEM, PENNSYLVANIA. 18015

U.S.A.

E-mail: CSQ0@LEHIGH.EDU 